University of Wollongong

Research Online

Faculty of Engineering and Information

Faculty of Engineering and Information

Sciences - Papers: Part B

Sciences

2014

\title{
An amorphous alloy core medium frequency magnetic-link for medium voltage photovoltaic inverters
}

Md Rabiul Islam

University of Technology Sydney, mrislam@uow.edu.au

Youguang Guo

University of Technology Sydney

Zhi Wei Lin

University of Technology Sydney, jacklin@eng.uts.edu.au

Jianguo Zhu

University of Technology Sydney

Follow this and additional works at: https://ro.uow.edu.au/eispapers1

Part of the Engineering Commons, and the Science and Technology Studies Commons

Research Online is the open access institutional repository for the University of Wollongong. For further information contact the UOW Library: research-pubs@uow.edu.au 


\title{
An amorphous alloy core medium frequency magnetic-link for medium voltage photovoltaic inverters
}

\begin{abstract}
The advanced magnetic materials with high saturation flux density and low specific core loss have led to the development of an efficient, compact, and lightweight multiple-input multiple-output medium frequency magnetic-link. It offers a new route to eliminate some critical limitations of recently proposed medium voltage photovoltaic inverters. In this paper, a medium frequency magnetic-link is developed with Metglas amorphous alloy 2605S3A. The common magnetic-link generates isolated and balanced multiple DC supplies for all of the $\mathrm{H}$-bridge inverter cells of the medium voltage inverter. The design and implementation of the prototype, test platform, and the experimental test results are analyzed and discussed. The medium frequency non-sinusoidal excitation electromagnetic characteristics of alloy $2605 \mathrm{~S} 3 \mathrm{~A}$ are also compared with that of alloy 2605SA1. It is expected that the proposed new technology will have great potential for future renewable power generation systems and smart grid applications.
\end{abstract}

\section{Keywords}

core, medium, frequency, magnetic-link, voltage, amorphous, photovoltaic, alloy, inverters

\section{Disciplines}

Engineering | Science and Technology Studies

\section{Publication Details}

M. Islam, Y. Guo, Z. Wei. Lin \& J. Zhu, "An amorphous alloy core medium frequency magnetic-link for medium voltage photovoltaic inverters," Journal of Applied Physics, vol. 115, pp. 17E710-1-17E710-4, 2014. 


\section{An amorphous alloy core medium frequency magnetic-link for medium voltage photovoltaic inverters}

Md. Rabiul Islam, Youguang Guo, Zhi Wei Lin, and Jianguo Zhu

Citation: Journal of Applied Physics 115, 17E710 (2014);

View online: https://doi.org/10.1063/1.4864050

View Table of Contents: http://aip.scitation.org/toc/jap/115/17

Published by the American Institute of Physics

\section{Articles you may be interested in}

A medium frequency transformer with multiple secondary windings for medium voltage converter based wind turbine power generating systems

Journal of Applied Physics 113, 17 A324 (2013); 10.1063/1.4795850

New Fe-based soft magnetic alloys composed of ultrafine grain structure Journal of Applied Physics 64, 6044 (1988); 10.1063/1.342149

Evolution of fcc $\mathrm{Cu}$ clusters and their structure changes in the soft magnetic $\mathrm{Fe}_{85.2} \mathrm{Si}_{1} \mathrm{~B}_{9} \mathrm{P}_{4} \mathrm{Cu}_{0.8}$ (NANOMET) and FINEMET alloys observed by X-ray absorption fine structure

Journal of Applied Physics 117, 17 A324 (2015); 10.1063/1.4916937

Magnetic properties of 120 -mm wide ribbons of high $\mathrm{B}_{\mathrm{S}}$ and low core-loss NANOMET ${ }^{\circledR}$ alloy

Journal of Applied Physics 117, 17B715 (2015); 10.1063/1.4913936

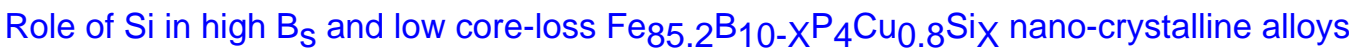
Journal of Applied Physics 112, 103902 (2012); 10.1063/1.4765718

Outstanding efficiency in energy conversion for electric motors constructed by nanocrystalline soft magnetic alloy "NANOMET ${ }^{\circledR}$ ", cores

AIP Advances 6, 055925 (2016); 10.1063/1.4944341

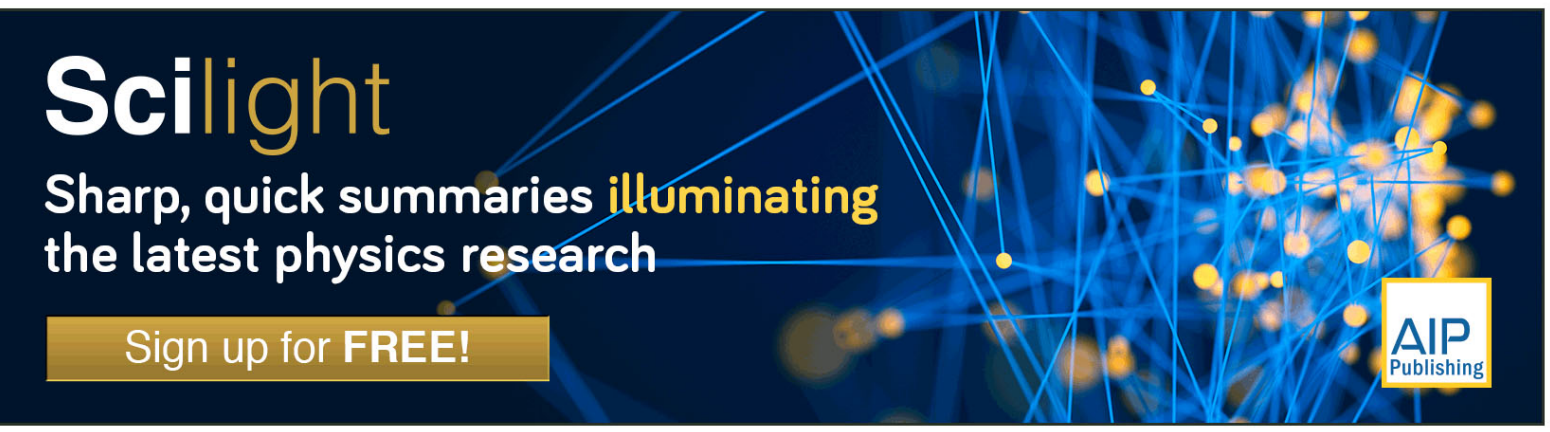




\title{
An amorphous alloy core medium frequency magnetic-link for medium voltage photovoltaic inverters
}

\author{
Md. Rabiul Islam, ${ }^{\text {a) }}$ Youguang Guo, Zhi Wei Lin, and Jianguo Zhu \\ Centre for Electrical Machines and Power Electronics, University of Technology Sydney, 15 Broadway, \\ Ultimo, NSW 2007, Australia
}

(Presented 7 November 2013; received 9 September 2013; accepted 4 November 2013; published online 6 February 2014)

\begin{abstract}
The advanced magnetic materials with high saturation flux density and low specific core loss have led to the development of an efficient, compact, and lightweight multiple-input multiple-output medium frequency magnetic-link. It offers a new route to eliminate some critical limitations of recently proposed medium voltage photovoltaic inverters. In this paper, a medium frequency magnetic-link is developed with Metglas amorphous alloy 2605S3A. The common magnetic-link generates isolated and balanced multiple DC supplies for all of the H-bridge inverter cells of the medium voltage inverter. The design and implementation of the prototype, test platform, and the experimental test results are analyzed and discussed. The medium frequency non-sinusoidal excitation electromagnetic characteristics of alloy 2605S3A are also compared with that of alloy 2605SA1. It is expected that the proposed new technology will have great potential for future renewable power generation systems and smart grid applications. (C) 2014 AIP Publishing LLC. [http://dx.doi.org/10.1063/1.4864050]
\end{abstract}

\section{INTRODUCTION}

For power transmission, a step-up transformer is usually used in the photovoltaic (PV) power plants to feed the solar energy to a medium voltage grid (e.g., $6-36 \mathrm{kV})$. The power transformers operated at frequencies of 50 or $60 \mathrm{~Hz}$ are heavy, large, and inefficient in many cases. For example, the volume and weight of a $0.4 / 36 \mathrm{kVA}, 1$ MVA vacuum cast coil transformer are about $4.3 \mathrm{~m}^{3}$ and $3250 \mathrm{~kg}$, and the no-load and full-load losses are $3.1 \mathrm{~kW}$ and $11.5 \mathrm{~kW}$, respectively. These penalties are critical in remote PV power plants, where the costs of installation and regular maintenance are high. Therefore, modular multilevel cascaded (MMC) medium voltage inverter for step-up-transformerless direct grid integration of PV systems has attracted a high degree of attention, ${ }^{1,2}$ since the commercial installation of medium/large-scale PV power plants in 2007. However, the MMC inverter requires multiple-isolated and balanced DC sources.

Compared with the power frequency transformers, the medium frequency (in the range of a few $\mathrm{kHz}-\mathrm{MHz}$ ) transformer-links have much smaller and lighter magnetic cores and windings, and thus, much lower costs. In 2012, multiple isolated medium-frequency-link based mediumvoltage inverter was proposed. ${ }^{1}$ In the proposed configuration, the voltage balancing is a challenging issue, since each $\mathrm{H}$-bridge cell is connected to a PV array through a DC/DC converter. Another common DC-link based approach was also proposed in $2012 .^{2}$ The design may reduce the voltage imbalance problem in the grid side. However, the generation of common DC-link voltage from different PV arrays makes the inverter operation complex and limits the range of maximum power point tracking (MPPT) operation.

\footnotetext{
a)Electronic addresses: Md.Islam@uts.edu.au and Rabiulbd@hotmail.com
}

In this paper, a common medium frequency magneticlink (instead of common DC-link) is proposed to minimize voltage imbalance problem with a wide range MPPT operation. The common magnetic-link generates isolated and balanced multiple DC supplies for all of the $\mathrm{H}$-bridge inverter cells of the medium voltage MMC inverter, which inherently overcomes the common mode and voltage imbalance problems. The elimination of common DC-link by common magnetic-link enables a wide range MPPT operation with independent controllability. To verify the feasibility of the new concept of common medium frequency magnetic-link based medium-voltage PV inverter, a scaled down $1.73 \mathrm{kVA}$ multiple-output medium frequency magnetic-link is developed. The link was tested experimentally with $1 \mathrm{kV}$ inverter system and satisfactory results were found. This paper presents the overall design and analysis of the proposed magnetic-link. The electromagnetic characteristics of alloy $2605 \mathrm{~S} 3 \mathrm{~A}$ are also compared with that of alloy $2605 \mathrm{SA} 1 .^{3}$

\section{ELECTROMAGNETIC DESIGN}

Taking into account the operating frequency of commercially available power semiconductor devices, a frequency window in the range of $1-20 \mathrm{kHz}$ is considered for the proposed system. Soft ferrites have high resistivity for medium/ high frequency applications, but the low saturation flux density (only $0.3-0.5 \mathrm{~T}$ ) limits their application in large power transmission. Silicon steel sheets have high saturation flux density for high power applications, but they cannot be used in medium/high frequency applications due to their low resistivity and eddy current loss. The amorphous alloy and nanocrystalline magnetic materials have excellent magnetic characteristics, such as high permeability, high saturation flux density, and relatively low core losses at medium/high frequency range. ${ }^{3-5}$ Two commercially available amorphous and 


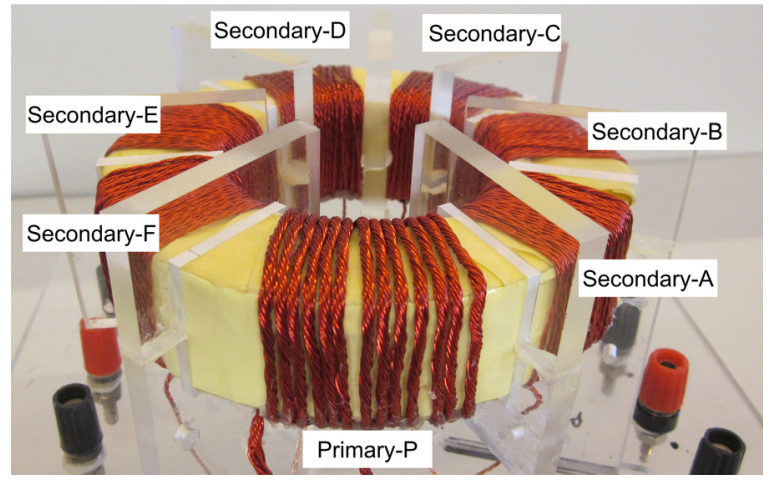

FIG. 1. A photo of the medium frequency magnetic-link.

nanocrystalline materials are Metglas and Finemet, which are manufactured by Hitachi Metals, Japan. Although, Finemet has lower specific core loss than Metglas, its saturation flux density (about $1 \mathrm{~T}$ ) is much lower than that of Metglas, which is up to $1.56 \mathrm{~T}$. Taking into account the flux density, specific core loss, and cost, the magnetic alloy 2605S3A has been chosen as a core material.

According to power inverter rating, the magnetic-link specifications, such as the rated power, frequency, excitation current, and voltage, are calculated. From the specifications of magnetic-link and data sheets of core materials, magneticlink initial parameters are calculated. These parameters are used as initial values of the optimization process. Different factors are considered during the design process, such as the winding dimensions, hole reserve for natural cooling, maximum temperature limits, maximum power loss, availability of core material stripe dimensions (standard available widths in the range of $2.5 \mathrm{~mm}-50 \mathrm{~mm}$ and thickness of $20 \mu \mathrm{m}$ ), parasitic parameters, skin and proximity effects, and possibility to induce identical voltage in multiple secondary windings. For simplicity of the winding process, a toroidal structure core is adopted. Finally, the inner and outer diameters, and height of the core were chosen as $6.5 \mathrm{~cm}$ and $10.5 \mathrm{~cm}$, and $25 \mathrm{~mm}$, respectively. Therefore, magnetic alloy 2605S3A stripe of $20 \mu \mathrm{m}$ thickness and $25 \mathrm{~mm}$ width is used to develop the core. The other parameters of this magnetic alloy are the impedance permeability at 1,6 , and $10 \mathrm{kHz}$ is about $0.0207,0.0199$, and $0.0196 \mathrm{H} / \mathrm{m}$, respectively, and the electrical resistivity, curie temperature, and saturation magnetostriction of magnetic alloy $2605 \mathrm{~S} 3 \mathrm{~A}$ is $138 \mu \Omega \mathrm{cm}, 358^{\circ} \mathrm{C}$, and $20 \mathrm{ppm}$, respectively.

The transformer has 14 turns for the primary winding and 25 turns for each secondary winding. To minimize the proximity effect, Litz wires are used for windings with single layer placement. The transformer has 13 and 3 insulated strands for primary and secondary windings Litz wires with a strand diameter of $0.4 \mathrm{~mm}$. The Metglas sheet was glued with Araldite on the surface of each layer, providing both the electrical insulation and mechanical bonding. The core volume and mass are $133.52 \mathrm{~cm}^{3}$ and $0.98 \mathrm{~kg}$. Figure 1 shows the photo of the final magnetic-link.

\section{EXPERIMENTAL INVESTIGATION}

In order to generate balanced multiple sources as required by the inverter, the voltage transformation ratios of all secondary windings versus the primary should be identical. The ratios were measured and they were found highly consistent with theoretical value, which is 1.781 with a variation of less than $\pm 0.08 \%$. The primary and secondary winding DC resistances were obtained at $0.0235 \Omega$ and $0.1630 \Omega$, and they were found highly consistent with the theoretical values, which are $0.0233 \Omega$ and $0.1620 \Omega$, respectively. The magnetic-link is excited by a medium frequency square wave primary voltage, which is generated by a Semikron compact insulated gate bipolar transistor module SK30GH123 (can be operated up to $20 \mathrm{kHz}$ ) based full-bridge inverter supplied by a DC voltage source.

From Faraday's law, the magnetic flux density, B, is calculated by measuring the open circuit terminal voltage of the pick-up coil (Secondary-D coil). The dependence of core loss on flux density was measured when the flux densities changed from $0.14 \mathrm{~T}$ to $1.3 \mathrm{~T}$; they were also compared with the core loss of magnetic alloy 2605SA1. The measured specific core loss of magnetic alloy $2605 \mathrm{~S} 3 \mathrm{~A}$ was $92 \mathrm{~W} / \mathrm{kg}$ at $10 \mathrm{kHz}$ square wave excitation of magnitude $0.73 \mathrm{~T}$ as shown in Figure 2, while the specific core loss of $140 \mathrm{~W} / \mathrm{kg}$ was measured with alloy 2605SA1 based magnetic-link. In comparison with alloy 2605SA1, 2605S3A shows about $35 \%$ less core loss at $10 \mathrm{kHz}$. The measured specific core loss of alloy $2605 \mathrm{~S} 3 \mathrm{~A}$ was $55 \mathrm{~W} / \mathrm{kg}$ at $6 \mathrm{kHz}$ square wave excitation of magnitude $0.73 \mathrm{~T}$, while the specific core loss of $69 \mathrm{~W} / \mathrm{kg}$ was measured with alloy 2605SA1. Compared with magnetic alloy 2605SA1, 2605S3A shows about 20\% less core loss at $6 \mathrm{kHz}$. Figure 3 shows the dependence of core loss on excitation frequency. It is observed that the specific core loss of alloy $2605 \mathrm{~S} 3 \mathrm{~A}$ is lower than that of $2605 \mathrm{SA} 1$ when the excitation frequency is above $750 \mathrm{~Hz}$.

From the Ampere's law, the field intensity, H, is calculated by measuring excitation current in the "Primary-P" coil. The primary winding was excited with different magnitude of currents at $12 \mathrm{kHz}$ excitation. The B-H loops are plotted based on experimental data with excitation currents of 3 and $5 \mathrm{~A}$, as shown in Figure 4. At $5 \mathrm{~A}$, the maximum flux density is about $0.8 \mathrm{~T}$ with field intensity of about $800 \mathrm{~A} / \mathrm{m}$, which satisfy the design requirements. It is also observed that the maximum flux density is stable for a temperature range of $23-120^{\circ} \mathrm{C}$. Figure 5 plots the B-H loops of magnetic alloy $2605 \mathrm{~S} 3 \mathrm{~A}$ at various temperatures. The B-H loops were also plotted at different frequencies, as shown in

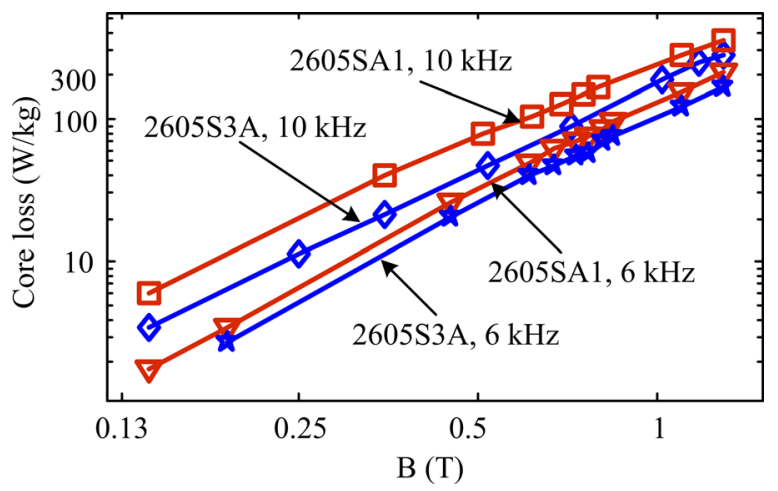

FIG. 2. Measured core losses at different flux densities ranging from $0.14 \mathrm{~T}$ to $1.3 \mathrm{~T}$. 


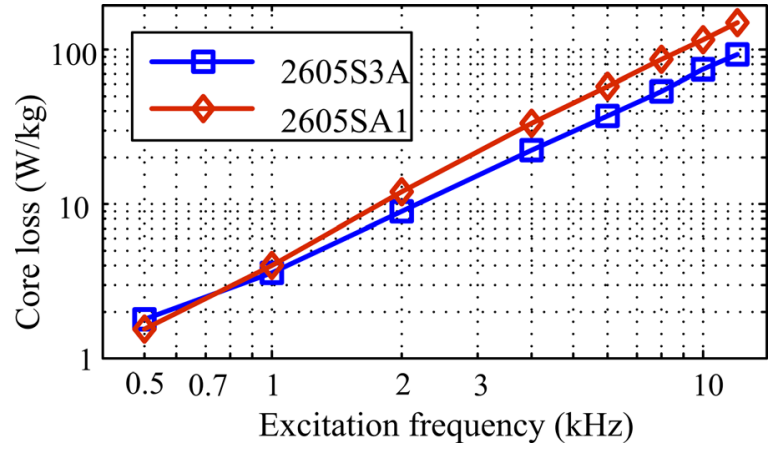

FIG. 3. Measured core losses at different excitation frequencies of $500 \mathrm{~Hz}$ to $12 \mathrm{kHz}$.

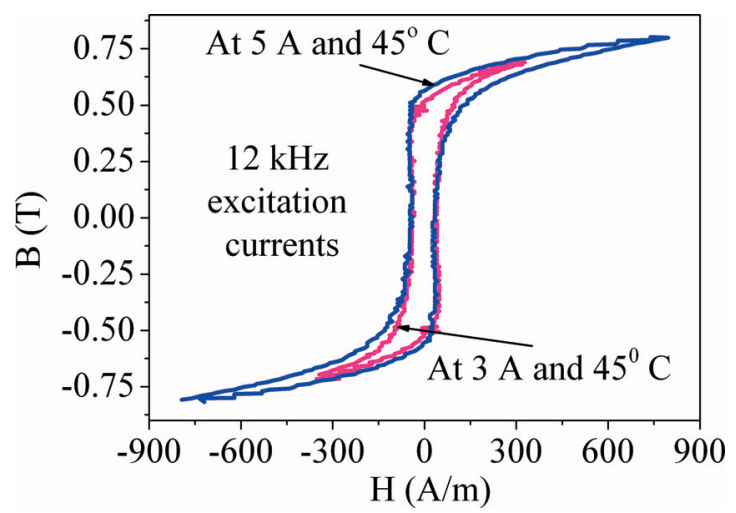

FIG. 4. Measured B-H loops at excitation currents of 3 and $5 \mathrm{~A}$.

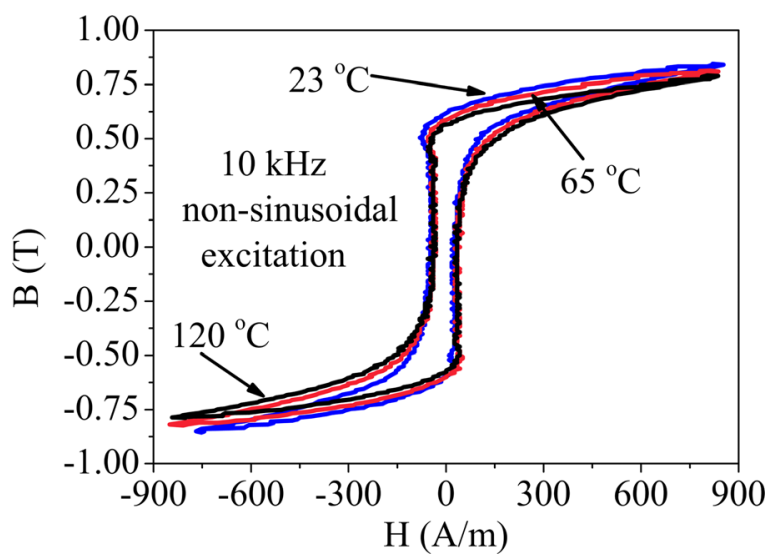

FIG. 5. Measured B-H loops of magnetic alloy 2605S3A at various temperatures ranging from $23^{\circ} \mathrm{C}$ to $120^{\circ} \mathrm{C}$.

Figure 6. The B-H loops of Metglas alloy 2605S3A are compared with that of Metglas alloy 2605SA1, as shown in Figure 7. In comparison with 2605SA1, the 2605S3A shows a slightly narrower B-H loop, i.e., lower core loss.

The total losses (core loss plus copper loss) measured from each secondary winding are very close to each other. For example, the total losses of all secondary winding A-B is $70.50,70.80,71.00,70.73,70.62$, and $71.02 \mathrm{~W}$, respectively, with a $0.5 \mathrm{~A}$ excitation current at $10 \mathrm{kHz}$. This is obligatory to generate balanced multiple sources. The six secondary windings were connected to a 3-phase 5-level inverter with an excitation frequency of $10 \mathrm{kHz}$. The output

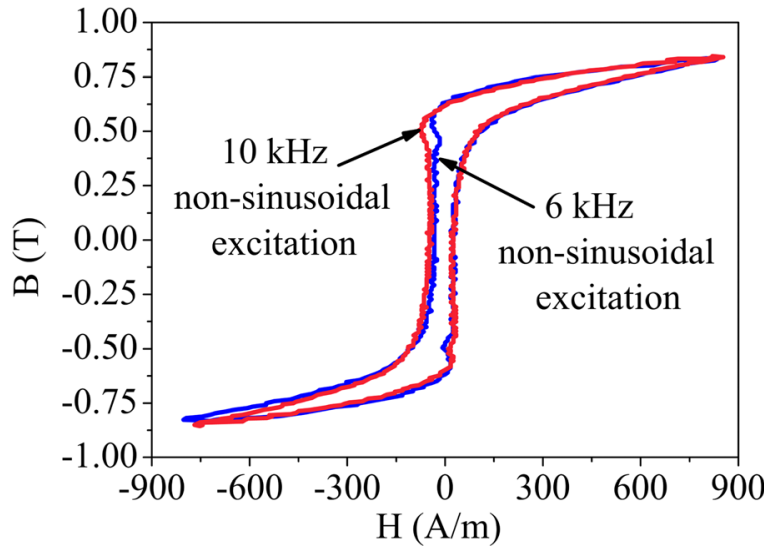

FIG. 6. Measured B-H loops of magnetic alloy 2605S3A at various excitation frequencies of 6 and $10 \mathrm{kHz}$.

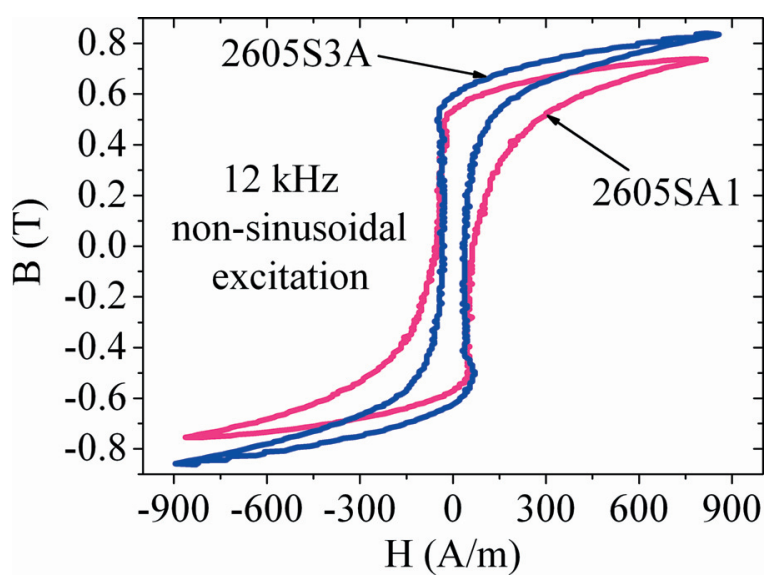

FIG. 7. Measured B-H loops at excitation current of $6 \mathrm{~A}$.

line voltage of prototype inverter was measured. The result demonstrates that the voltages from the magnetic-link are balanced, and it overcomes the limit of MPPT operation.

\section{CONCLUSION}

A magnetic-link using amorphous magnetic alloys is proposed to generate isolated and balanced multiple DC supplies for all of the H-bridge inverter cells of the medium voltage MMC inverter. It overcomes common mode and voltage imbalance problems. Two alloys, 2605S3A and 2605SA1, were used in the study, and their B-H loops and specific core losses were analysed. The results show that magnetic alloy 2605S3A has a slightly narrower B-H loop and lower specific core loss at the frequency between 1 and $20 \mathrm{kHz}$ than that of alloy $2605 \mathrm{SA} 1$ and is suitable for the magnetic-link for medium voltage inverter.

${ }^{1} \mathrm{H}$. Choi et al., in Proceedings of the 3rd IEEE International Symposium on Power Electronics for Distributed Generation Systems, Aalborg, Denmark, 25-28 June, 2012, pp. 801-807.

${ }^{2}$ S. Rivera et al., in Proceedings of the 3rd IEEE International Symposium on Power Electronics for Distributed Generation Systems, Aalborg, Denmark, 25-28 June, 2012, pp. 690-697.

${ }^{3}$ M. R. Islam et al., J. Appl. Phys. 113, 17A324 (2013).

${ }^{4}$ Z. Zhang et al., J. Appl. Phys. 112, 103902 (2012).

${ }^{5}$ A. Makino et al., J. Appl. Phys. 109, 07A302 (2011). 